\title{
Protein pathway biomarker analysis of human cancer reveals requirement for upfront cellular-enrichment processing
}

\author{
Alessandra Silvestri ${ }^{1,2}$, Alfonso Colombatti ${ }^{2}$, Valerie S Calvert ${ }^{1}$, Jianghong Deng ${ }^{1}$, Enzo Mammano ${ }^{3}$, Claudio Belluco ${ }^{4}$, \\ Francesco De Marchi ${ }^{4}$, Donato Nitti ${ }^{3}$, Lance A Liotta ${ }^{1}$, Emanuel F Petricoin ${ }^{1}$ and Mariaelena Pierobon ${ }^{1}$
}

Tissues are complex structures composed of different cell types, each of which present specific functions and characteristics. To better understand and measure the effect of tumor cell enrichment on protein pathway profiling and drug target activation measurements, the signaling activation portraits of laser capture microdissected (LCM) cancer epithelium and tumor stroma were compared with patient-matched whole-tissue specimens from 53 primary colorectal cancer samples. Microdissected material and whole-tissue lysate from contiguous cryostat sections were subjected to reverse-phase protein microarray analysis to determine the level of phopshorylation and expression of 75 different proteins known to be involved in cancer progression. The results revealed distinct differences in the protein activation portraits of cancer epithelium and stroma. Moreover, we found that the signaling activation profiles of the undissected whole-tissue specimens are profoundly different from the matched LCM material. Attempts to rescale the undissected pathway information based on percent endogenous tumor epithelium content were unsuccessful in recapitulating the LCM tumor epithelial signatures. Analysis of epidermal growth factor receptor phosphorylation and COX2 expression in these same sample sets revealed wholesale differences in the rank ordering of patient determination when LCM was compared with undissected samples. On the basis of these data, we conclude that accurate protein pathway activation status, which is under evaluation as a basis for patient selection and stratification for personalized therapy, must include upfront cellular-enrichment techniques such as LCM to generate accurate drug target activation status.

Laboratory Investigation (2010) 90, 787-796; doi:10.1038/labinvest.2010.47; published online 1 March 2010

KEYWORDS: LCM; proteomic analysis; reverse-phase protein microarray; signal transduction; therapeutic stratification; tissue heterogeneity

Tissues are complex structures composed of different cell types. Diseased cells arise in context of the complex tissue microenvironment and are influenced by the complex interactions between multiple cell populations, including epithelium, stroma immune cells, vasculature and nerve cells. During the neoplastic progression, tumor cell interactions with cells in the host microenvironment influence virtually all aspects of tumor growth, survival, local invasion and distant dissemination. ${ }^{1}$ Cancer-associated fibroblasts, endothelial cells and macrophages are thought to promote cancer progression in some cases. ${ }^{2}$ Stromal adaptation surrounding the tumor may result in the elaboration of cytokines and chemokines that drive invasion through chemotaxis and haptotaxis. ${ }^{3-5}$
The study of phosphorylated, cleaved, glycosylated and ubiquinated proteins has provided fundamental information for the understanding of cancer progression. Critical cell signaling pathways, often aberrantly activated in cancer, are driven largely by phosphorylation changes in key signaling molecules. Modulation of deranged kinase activities that underpin tumorigenesis and metastasis promises to yield new targets for novel molecularly targeted therapeutics. ${ }^{6-9}$ Thus, it is of critical importance to identify and characterize ongoing cell signaling network deregulations within the tissue microenvironment in clinical research tissue samples.

To understand the molecular changes occurring in specific tissue subpopulations, it is necessary to separate and analyze

\footnotetext{
${ }^{1}$ Center for Applied Proteomics and Molecular Medicine, George Mason University, Manassas, VA, USA; ${ }^{2}$ Department of Experimental Oncology 2, CRO-IRCSS, National Cancer Institute, Aviano, Italy; ${ }^{3}$ Clinica Chirurgica II, Department of Oncological and Surgical Sciences, University of Padova, Padova, Italy and ${ }^{4}$ Department of Surgical Oncology, CRO-IRCSS, National Cancer Institute, Aviano, Italy

Correspondence: Dr M Pierobon, MD, Center for Applied Proteomics and Molecular Medicine, George Mason University, 10900 University Blvd, MS 1A9, Bull Run Hall, Room 325, Manassas, VA 20110, USA.
}

E-mail: mpierobo@gmu.edu

Received 17 September 2009; revised 22 October 2009; accepted 21 December 2009 
each cell subpopulations. Recent analyses of tumor and stromal signaling changes have revealed important epithelialmesenchymal transition signaling alterations in tumor epithelium. ${ }^{10}$ These types of complex tissue microenvironment interactions within different compartments of the tissue milieu may not be accurately recapitulated in cell culture. Consequently, direct molecular analysis of ongoing signaling cascades and networks within human tissue subpopulations is necessary for a more full understanding of human cancer pathogenesis. Nevertheless, a key barrier to understand signaling changes within the context of human clinical material is the heterogeneity of the cellular composition of the tissue itself and the need to separate molecular alterations within the different stromal, epithelial and immune cell compartments within the tumor.

The introduction of laser capture microdissection (LCM) in the late 1990s represented a novel way to overcome the limitations related to tissue heterogeneity. LCM technology uses an inverted microscope that allows the operator to directly visualize the cells of interest and to isolate them from the surrounding microenvironment. In the last decade, by combining LCM to RNA/DNA and protein microarrays, immunoassays and mass spectrometry analysis, large-scale genomic and proteomic analysis has been generated with highly specific results. ${ }^{1-13}$ The effective coupling of LCM with downstream high throughput molecular assays that are able to simultaneously analyze and measure hundreds of clinical samples and end points with high reproducibility have been introduced. One of these techniques that has shown great utility, especially for multiplexed cell signaling analysis from clinical material, is the reverse-phase protein microarray (RPMA) platform. ${ }^{14-17}$ Unlike immunohistochemistry, RPMA is a technique that permits the simultaneous analysis of hundreds of samples allowing feasible direct comparison between patients and pathological conditions. RPMA also avoids subjective data interpretation and does not encounter antigen retrieval treatment difficulties.

As we enter the era of individualized therapy, in which administration of targeted therapies are based on the patientspecific molecular portrait of ongoing signaling activity, it is of absolute critical importance that the molecular information used to stratify and select therapies is correct. A current ongoing debate is the requirement for techniques such as LCM in the overall workflow of molecular diagnostics. Although recent data in breast cancer have yielded clues as to the criticality of LCM in producing accurate protein signal mapping analysis, ${ }^{18}$ a detailed analysis of signal pathway mapping information obtained from patient-matched undissected and dissected tumor and stromal cell compartments has not been performed. This type of analysis is critical in providing guidance to scientists on the impact of LCM on pathway analysis. In this study, we use LCM and RPMA to compare the protein-signaling profiles obtained by enriched cell populations isolated from human colon cancer specimens as a case study for analysis. For this purpose, highly enriched cancer and stromal cell population were separately captured by LCM. Fibroblast, stromal fiber and rare lymphocytes were isolated as stromal population. The signal pathway activation portrait of the enriched populations was compared with patient-matched whole-tissue undissected profiles. Contiguous cryostat slides were immediately lysed and subjected to protein analysis. RPMA-based measurements of 75 different total, phospho and cleaved key signaling proteins known to be involved in cell survival, migration, apoptosis and differentiation was performed to provide a broad-scale basis of signaling activation states to compare undissected $v s$ LCM network maps. Furthermore, to more fully evaluate the need for LCM to produce accurate pathway data and ascertain whether pathway knowledge of the tumor epithelium could be recapitulated without having to perform more timeintensive methods such as LCM, we attempted to mathematically rescale the protein array data from the undissected material based on knowledge of percent tumor epithelium from each specimen. The pathway maps and individual protein-signaling data obtained from LCM-procured purified tumor and stromal compartments were then compared with the rescaled undissected material.

\section{MATERIALS AND METHODS Tissue Samples}

A total of 53 primary CRC samples collected during surgical procedure at the Clinica Chirurgica II in Padua (Italy) were enrolled in this study. Each sample was collected according to institutional review board-approved protocol and immediately snap frozen. Certified pathologists performed histopathological diagnosis on the entire study set and confirmed the malignant nature of the lesions.

As tissues are a heterogeneous cellular mixture in which different cellular subtypes varies significantly from patient to patient, we separately analyzed the signal generated by highly enriched tumor epithelium and the surrounding stromal tissue to identify possible mathematical manipulations that are able to recapitulate the neoplastic epithelium activity by analyzing the whole tissue. For this purpose, highly enriched cancer cell population and stroma component were separately isolated using LCM, whereas contiguous cryostat slides were immediately lysed to analyze the signal generated by the whole tissue.

\section{Tissue Microdissection and Cell Lysis}

Each sample was subjected to LCM to obtain highly enriched epithelial cancer and stroma cell population under the guidance of a board certified pathologist (LL). Frozen tissue sections ( $8 \mu \mathrm{m}$ thick) were fixed in $70 \%$ ethanol, stained with hematoxylin and dehydrated in 70, 95 and 100\% ethanol, followed by xylene and then air dried. Ethanol (70\%), deionized water and hematoxylin were supplemented with Complete Mini protease inhibitor tablets (Roche Applied Science, Indianapolis, IN, USA). For each sample $\sim 20000$ epithelial cancer cells and $\sim 20000$ stroma cells were microdissected with Pixcell II Laser Capture Microdissection 
System (Arcturus, Mountain View, CA, USA) and stored on microdissection caps (CapSure Macro LCM Caps, Arcturus) at $-80^{\circ} \mathrm{C}$. Microdissected material as well as contiguous cryostat sections were lysed at the concentration of 600 cells per $\mu$ l with extraction buffer- $2.5 \%$ of $\beta$-mercaptoethanol in a 1:1 solution of Tris-Glycine SDS Sample Buffer (Invitrogen, Life Technologies, Carlsbad, CA, USA) and Tissue Protein Extraction Reagent (Pierce, Rockford, IL, USA)—for $5 \mathrm{~min}$ at room temperature. The cellular lysates were boiled for $8 \mathrm{~min}$ and stored at $-80^{\circ} \mathrm{C}$.

\section{Lysate Printing and Protein Staining}

Using the 2470 Aushon Arrayer (Aushon BioSystems, Billerica, MA, USA) outfitted with $350-\mu \mathrm{m}$ pins, sample lysates were printed on two sets of 100 nitrocellulose coated slides (Whatman, Sanford, ME, USA) and subjected to RPMA analysis. Each sample was printed in duplicate and in twopoint dilution curves. Slides 25, 50, 75 and 100 of each set were then stained with Sypro Ruby Protein Blot Stain (Molecular Probes, Eugene, OR, USA) and visualized with NovaRay Image Acquisition Software (Alpha Innotech, San Leandro, CA, USA) to determine the total protein concentration. Before antibody staining, the slides were treated with Reblot antibody stripping solution (Chemicon, Temecula, CA, USA), and incubated $5 \mathrm{~h}$ at room temperature in blocking solution with constant shaking. Blocked slides were stained with 75 different antibodies targeting total and phosphorylated proteins using an automated stainer (Dako Cytomation, Carpinteria, CA, USA). Each antibody was subjected to rigorous validation for single band specificity at the correct MW by Western blotting along with the use of appropriate ligand-induction controls for phospho-specific antibodies. Catalyzed Signal Amplification System kit (Dako Cytomation, Carpinteria, CA, USA) and fluorescent IRDye 680 Streptavidin (LI-COR, Lincoln, Nebraska, USA) were used as detection system. Stained slides were scanned with NovaRay Image Acquisition Software (Alpha Innotech).

\section{Data Analysis}

Acquired images of each slide were analyzed using MicroVigene software (Vigenetech, Carlisle, MA, USA), which finds spots, performs local background subtraction, averages replicates and normalizes each sample for the total protein value. Normalization between slides was performed using control cell lysate printed on each slide as a bridging case.

The percentage of carcinoma cells in each sample was histopatologically determined and it was calculated considering the average value between two independent analyses (difference between the two analysis was $<5 \%$ ) (Table 1 ).

To determine whether it is possible to recapitulate the contribution of the cancer cell profile by using the wholetissue lysate, each sample was subjected to multiplication of the intensity value of the whole-tissue lysate for the cancer percentage. RPMA values obtained from LCM-procured cancer epithelium, stroma and whole-tissue lysate were

\section{Table 1 Sample classification considering percentage of cancer epithelium}

Percentage of cancer epithelium

Number of samples

$\begin{array}{rr}10 & 3 \\ 20 & 10 \\ 30 & 11 \\ 40 & 9 \\ 50 & 11 \\ 60 & 6 \\ 70 & 3\end{array}$

The percentage of cancer epithelium present in each sample was histopathologically determined by two separate analyses. The average value between two independent operators, each overseen by a board-certified pathologist, was used (difference between the two analysis was $<5 \%$ ).

subjected to unsupervised clustering analysis, performed using JMP 5.1 software (SAS, Cary, NC, USA). To compare the data obtained from the different components, the intensity values of LCM cancer epithelium (CE-LCM), LCM stroma (CS-LCM), whole-tissue (WhT) and extrapolated cancer epithelium $(\mathrm{EV}=\mathrm{WhT}$ intensity value $\times \%$ cancer epithelium) were analyzed with Excel software to calculate linear correlation that allowed us to compare data obtained from LCM and whole-tissue material.

\section{RESULTS \\ Pathway Activation Mapping of Patient-Matched Undissected and LCM-Procured Stroma and Epithelial Cells}

To investigate pathway activation similarities between whole undissected tissue and LCM-procured tumor epithelium and tumor stroma cells, protein-signaling mapping was performed using RPMA data generated from 75 end points, which comprise signaling proteins known to be involved in tumorigenesis and metastatic progression. Approximately 20000 epithelial cancer cells and 20000 stroma cells were isolated from each sample using LCM from contiguous cryostat sections to minimize the heterogeneity of cell representation in the tissue. Two-way unsupervised hierarchical clustering analysis was used to broadly compare the profile of highly enriched cancer epithelium (CE-LCM), whole-tissue lysates (WhT) and stromal compartment (CS-LCM) by generating separate heatmaps (Figures 1a and b, and Figure 2a), as well as a heatmap (Supplementary Figure S1) in which all three components were comingled (LCM stroma, LCM epithelium and undissected material from the same patient). The results show that the pathway profiles of the epithelium and stromal components are very different, with patient and signaling end point associations entirely different between the two. This was even more evident when the larger clustering analysis was performed 


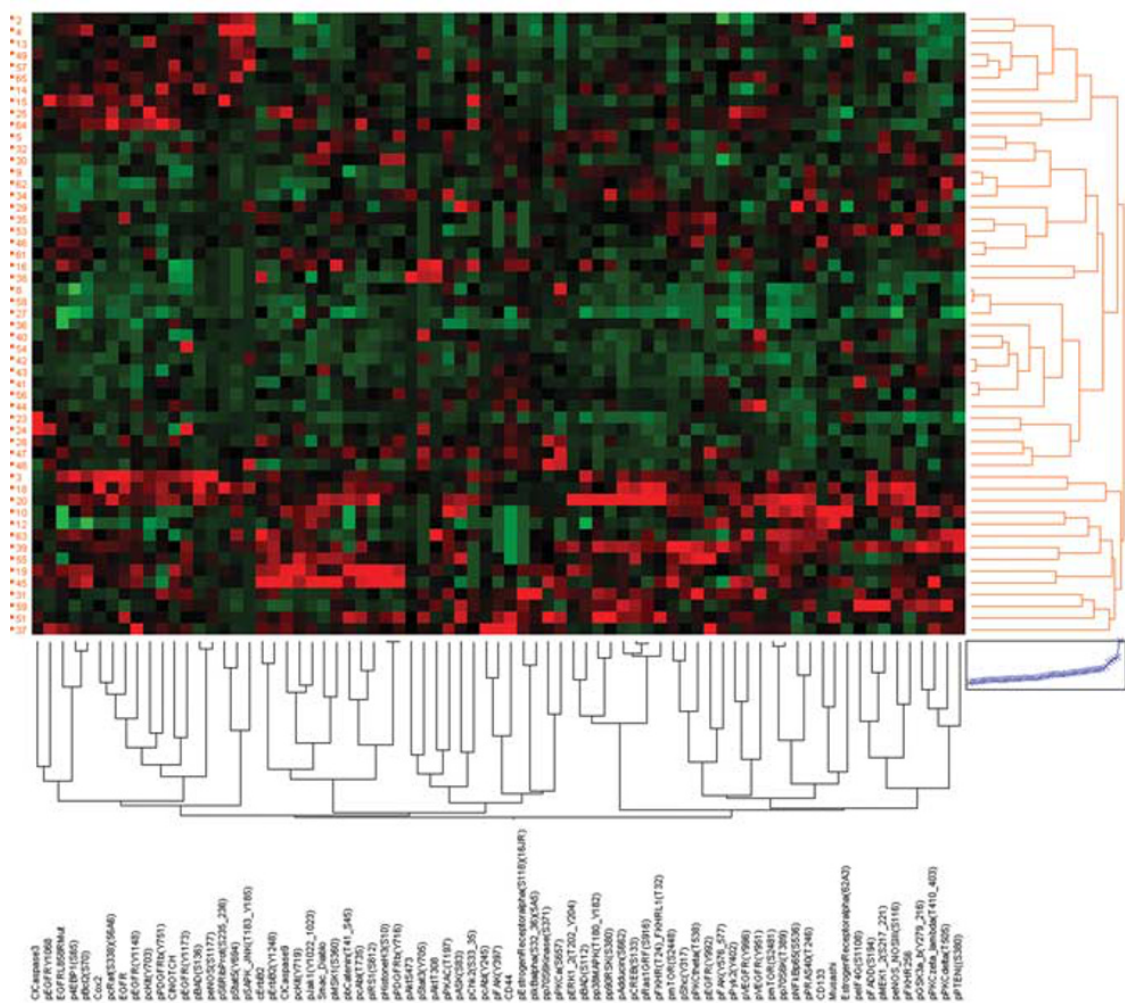

b

\section{WhT}
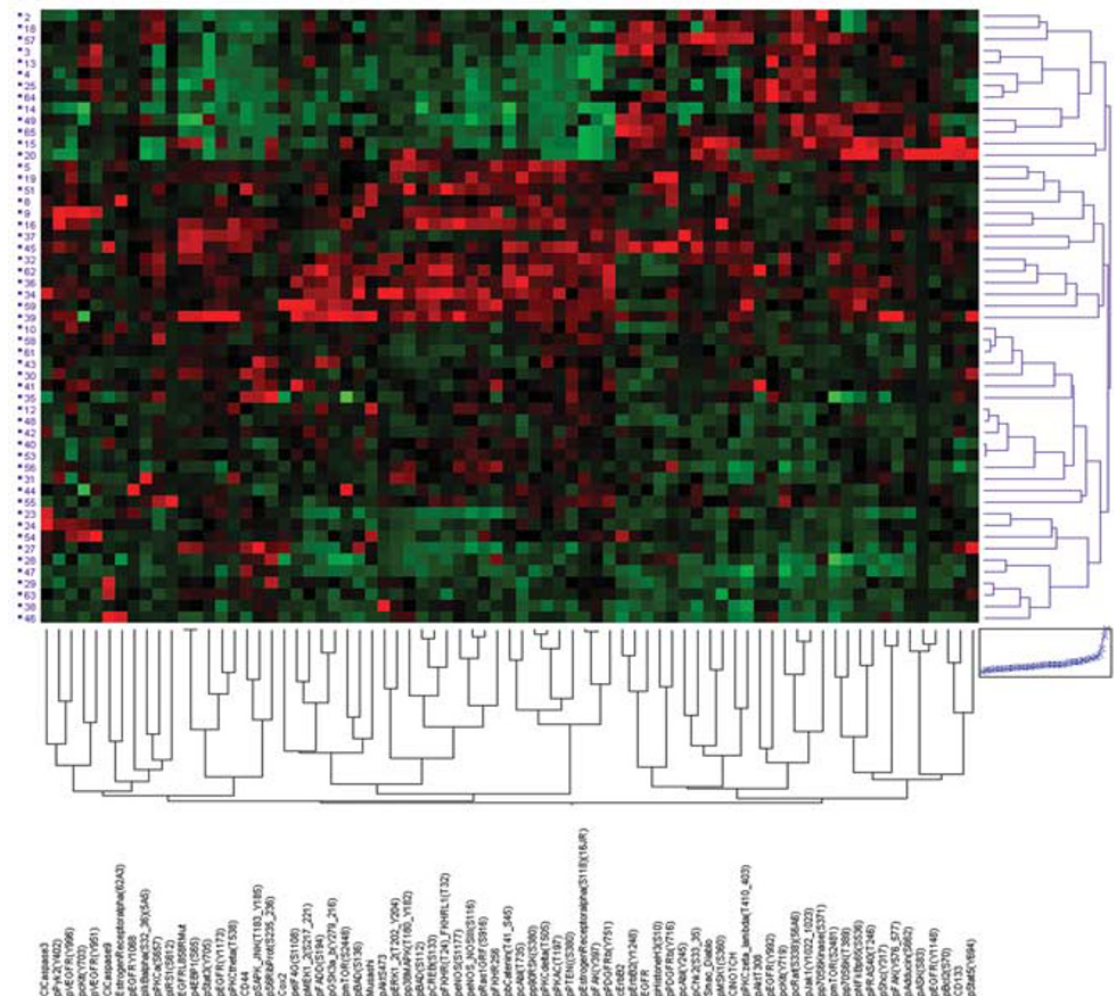

Figure 1 Two-way unsupervised hierarchical clustering of laser capture microdissected (LCM) tumor epithelium, and patient-matched undissected material. Relative intensity values of 75 different signaling proteins obtained by reverse phase protein microarray were used. (a) LCM-procured cancer epithelium (CE-LCM); (b) patient-matched undissected whole tissue (WhT). Red indicates a high relative level of protein activation or expression, black for median value and green indicates low relative level of protein activation or expression. 


\section{a}

CS-LCM


Figure 2 Two-way unsupervised hierarchical clustering of laser capture microdissected (LCM) cancer stroma and extrapolated value. Relative intensity values of 75 different signaling proteins obtained by reverse-phase protein microarray were used. (a) LCM-procured cancer stroma (CS-LCM);

(b) extrapolated value $(\mathrm{EV})(\mathrm{EV}=\mathrm{WhT}$ intensity value $\times \%$ cancer epithelium). Red indicates a high relative level of protein activation or expression, black for median value and green indicates low relative level of protein activation or expression. 

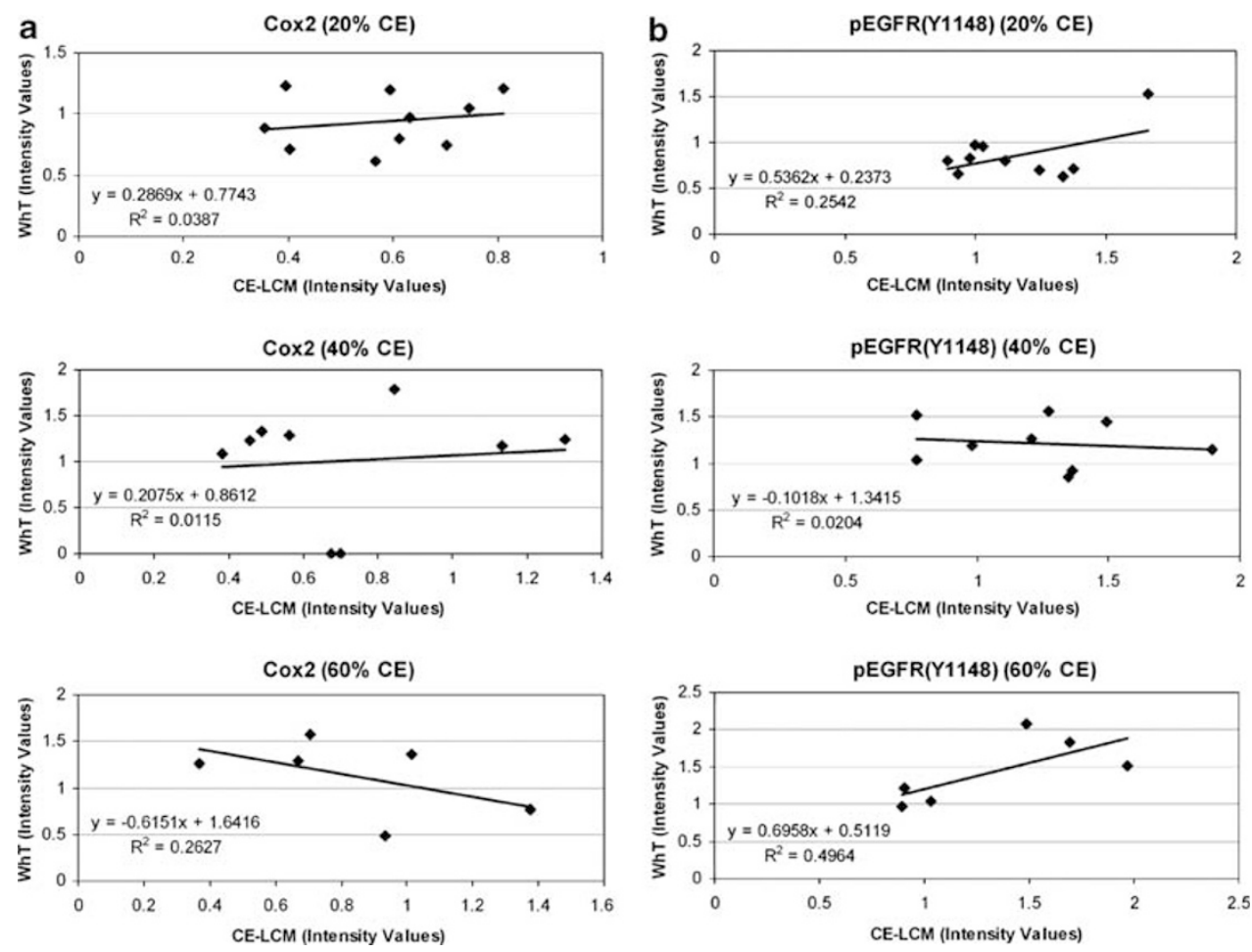

Figure 3 Two-way scatter plots of Cox-2 and phosphorylated EGFR from LCM cancer epithelium vs patient-matched whole-tissue intensity values. Relative levels of Cox-2 (a) and pEGFR Y1148 (b) from RPMA data obtained from patient-matched undissected ( $y$ axis) and LCM-procured epithelium ( $x$ axis) were analyzed as a two-way scatter plot to compare the relative intensity values from those two inputs. Correlation of whole-tissue and LCM cancer epithelium in tissues is shown with different starting percentages of cancer epithelium in the tissue specimen: $20 \%$ tumor epithelium (top panel); $40 \%$ tumor epithelium (middle panel); and 60\% tumor epithelium (bottom panel). Trend line along with $R^{2}$ value is shown for each graph.

(Supplementary Figure S1). Indeed, the samples break up in smaller clusters according to similar processed tissue.

These results clearly reveal that signaling portraits of the undissected material are entirely different than both the stroma and epithelial signatures even from the same patient sample.

We next attempted to recapitulate the signatures attained from the LCM epithelium from the undissected material by multiplying the intensity values of the undissected sample by the percent tumor epithelium from each patient sample (extrapolated value, EV). The results from this manipulation are shown in Figure $2 \mathrm{~b}$, and reveal that this manipulation does not recapture the end point or patient associations seen in the LCM epithelium heatmap (Figure 1a).

Recent studies have found that LCM procedure itself does not affect protein expression or phosphorylation levels. ${ }^{19,20}$ Thus, the differences observed here arise from the input sample and not process differences. Previous study has revealed that microdissection of $>1000$ cells produces consistent results between cells populations within the same specimen, hence these differences are not expected to be due to cellular variability. ${ }^{14,21,22}$

\section{Impact of Cellular Enrichment on Individual Protein-Signaling Molecule Measurements}

On the basis of these results, which indicate that pathway maps from undissected material do not recapitulate the tumor or stroma signatures from the LCM purified patientmatched samples, we next sought to focus more specifically on two important analytes that are thought to have a central role in colorectal cancer progression and treatment: COX2 and epidermal growth factor receptor (EGFR). Specifically, we evaluated phosphorylated EGFR (pEGFR Y1148) as this end point would more directly measure the level of receptormediated signaling itself. The RPMA-generated intensity values of these two analytes from patient-matched LCMprocured cancer epithelium and undissected tissue were directly compared using two-way scatter plots. Moreover, within this context and to evaluate the impact of the starting tumor percentages on the concordance of protein expression values obtained from undissected material $v s$ LCM cells, we analyzed the correlation of undissected $v s$ LCM measurements of COX2 and pEGFR in tumor specimens with low starting tumor epithelial content (20\%), medium (40\%) and higher $(60 \%)$ tumor cell populations. For each sample, the percentage of cancer epithelium was determined by two independent operators through the evaluation of hematoxylin and eosin-stained slides. The average value of the two independent histopatological analyses was used. The results shown in Figure 3 reveal little to no correlation between the undissected tissue values of COX2 and phosphorylated EGFR and the values of these two analytes obtained from the LCM tumor epithelium from the same patient. These results were 
a

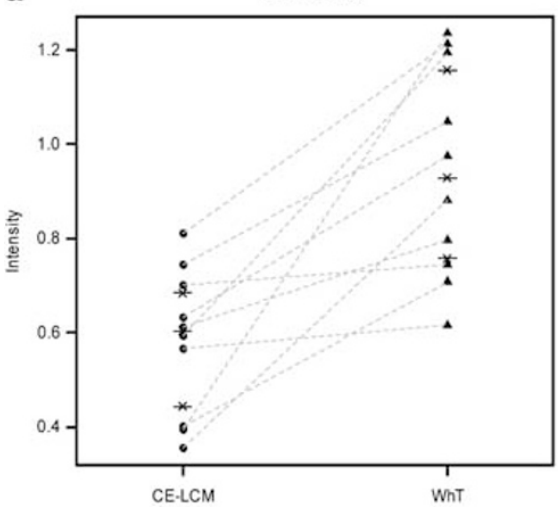

b

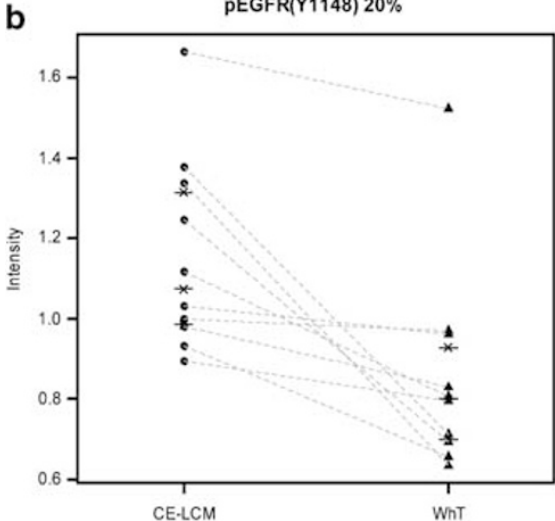

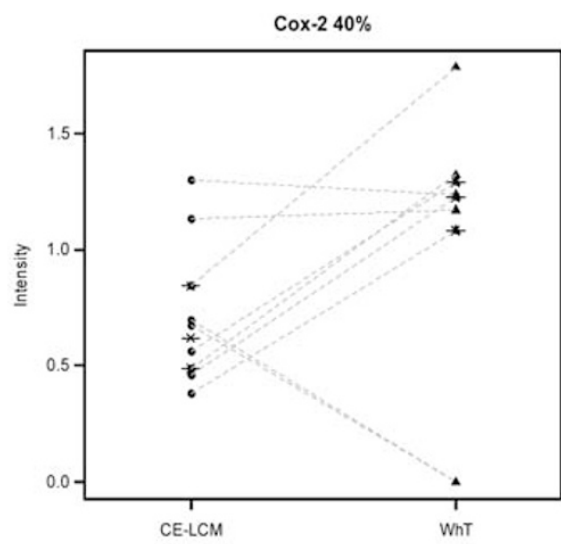
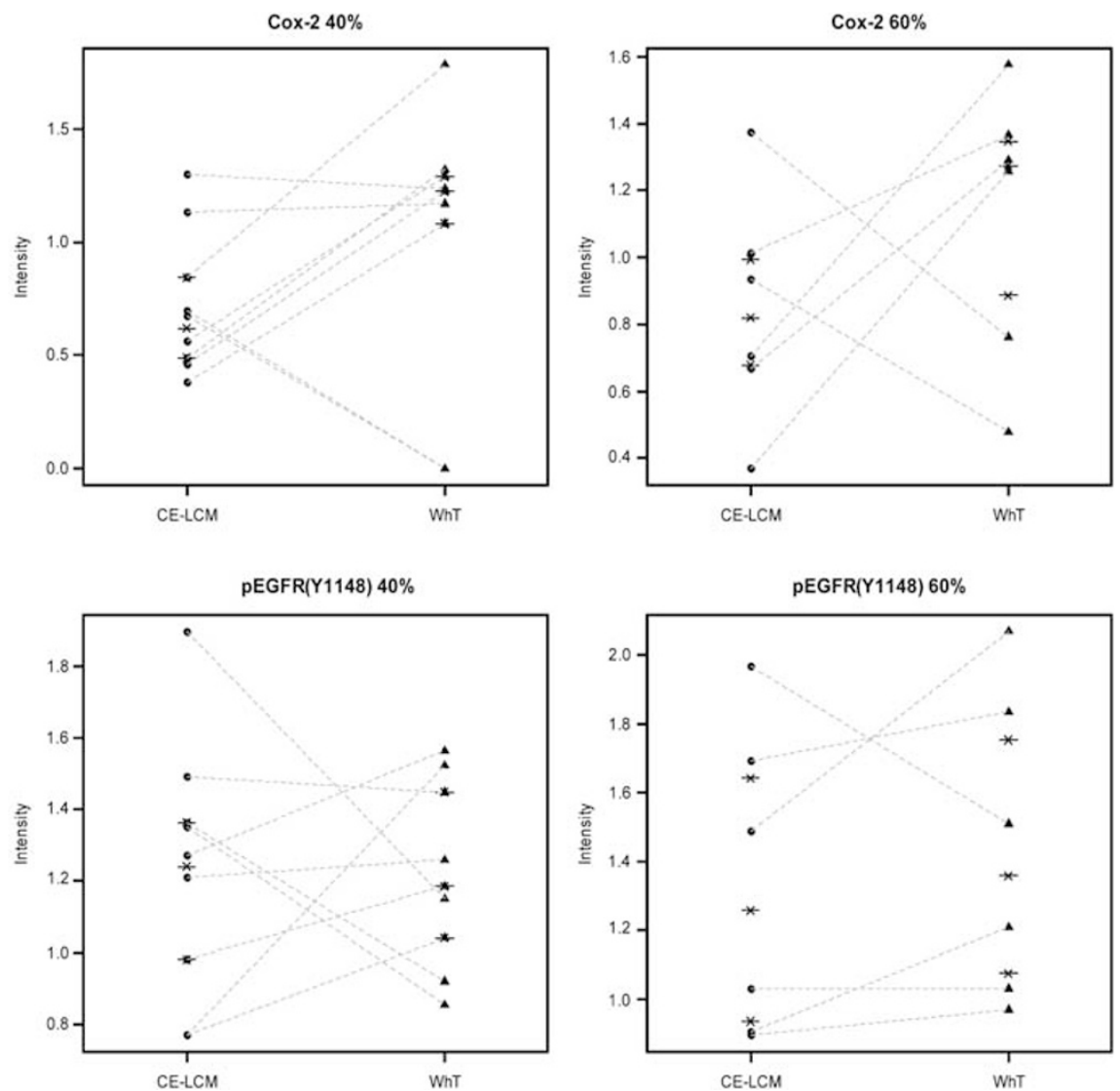

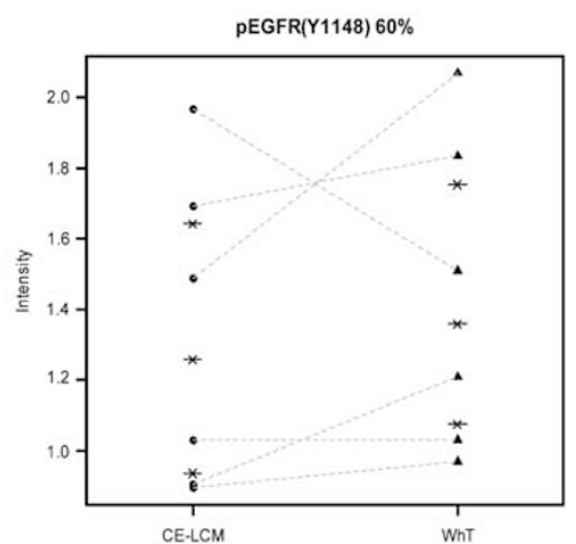

Figure 4 Rank-order plots comparing (a) COX2 protein expression and (b) phosphorylated EGFR (Y1148) levels. RPMA-generated relative intensity values are plotted for undissected (WhT) and laser capture microdissected tumor epithelium (CE-LCM) for both COX2 expression and phosphorylated EGFR (Y1148) levels. Comparisons are made with three differing levels of starting endogenous tumor epithelium (20, 40 and $60 \%)$. Changes in rank ordering are shown with dotted lines connecting the matched patient samples. Quartiles are designated by horizontal lines in each plot.

consistent even when tissues with larger starting endogenous percentages of tumor epithelium were used, which indicates that protein measurement results obtained even from tissue specimens that are majority tumor epithelium may not accurately reflect the level of the protein in the highly enriched tumor epithelium population. Thus, the level of proteins and phosphorylated proteins within the different tissue components is variable and not theoretically determinable a priori, and therefore it is not possible to extrapolate the contribution of every tumor compartment to the data output.

As most of the new molecularly targeted inhibitors are protein kinase and signaling inhibitors, an exciting aspect of protein-signaling analysis of human tissue is the potential for this method to become a companion diagnostic assay that can select and stratify patients for therapy based on the inherent molecular activity of the drug target proteins. Thus, a critical component of this workflow is the accurate determination of the relative or quantitative levels of the pathway biomarkers used for stratification. Consequently, we next examined whether the relative rank order levels determined for COX2 and phosphorylated EGFR, two important drug targets for colorectal cancer, would significantly change if the cellular input for RPMA analysis was from an undissected or the patient-matched microdissected sample. We analyzed these comparisons using a study set of colorectal tumor samples with differing amounts of endogenous tumor epithelium so that any rank order changes could be understood in the context of the amount of tumor in the undissected whole-tissue sample. Three groups of colorectal patients that had the same approximate endogenous starting amounts of tumor epithelium (10 patient samples with $20 \%$ tumor epithelium, 9 patient samples with $40 \%$ tumor epithelium and 6 patient samples with $60 \%$ tumor epithelium) were used for this analysis. The rank order of the undissected to LCM cohort within each group was compared to highlight whether the relative patient position was maintained in samples with the same starting cancer percentage, and whether or not there was any trend in concordance of ranking as tissue tumor epithelium percentage increased. As shown in Figure 4 , in which scatter plots of the relative intensity values of the RPMA data for the COX2 (Figure 4a) and phosphorylated EGFR (Y1148) (Figure 4b) are shown for both LCM (CE-LCM) and patient-matched undissected tissue (WhT), regardless of the starting tumor percentage of the tissue, we observed large changes in the rank ordering of the patient samples if the analysis emanated from undissected 

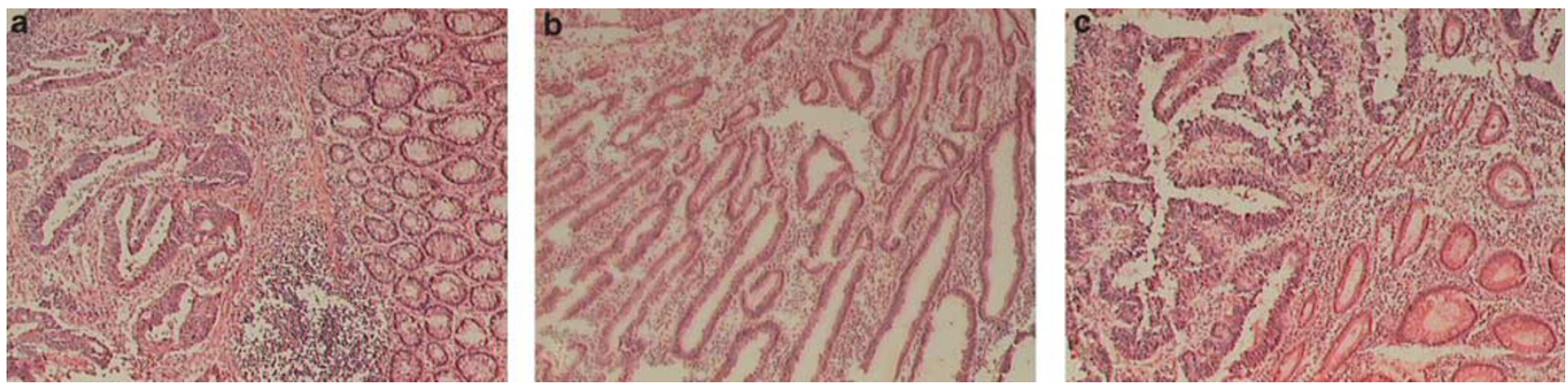

Figure 5 Tissue histology analysis. (a, b and $\mathbf{c}$ ) Three different samples with same amount of cancer epithelium were considered to highlight the strong difference in tissue composition between specimens.

material was compared with the one obtained using LCM material, with little concordance between the samples. These results indicate the dramatic differences in how a final determination of a specific drug target activity or expression level, potentially used to select and stratify patients for therapy, could be effected by starting with undissected material instead of enriched tumor cells, even from patients with the same relative amount of starting tumor epithelium in the whole-tissue specimen.

\section{DISCUSSION}

The identification of protein pathway biomarkers that are themselves the targets for molecular inhibitors is becoming a critical aspect to the development of the so-called 'companion diagnostics' in oncology, which would lead to selection and stratification of patients for specific therapies based on the underpinning molecular portrait of each patients tumor. New types of proteomic technologies, such as the RPMA, provide the ability to quantitatively measure the activity of hundreds of protein drug targets at once from a tiny needle biopsy specimen. However, given the central 'gate-keeping' potential importance of these companion diagnostic analytes, it is imperative that they accurately reflect the level of activity or expression of the drug target that is used for therapeutic selection. Indeed, it could be argued that there is no more important role for a biomarker than to guide therapeutic selection and patient stratification for therapy. Consequently, we sought to investigate the impact that more laborious upfront cellular-enrichment techniques such as LCM have on the accurate determination of drug target expression/activation. Previous studies have indicated that LCM process itself has no measurable impact in protein expression or activation levels measurements when specific protocols are followed. ${ }^{14,19,20}$ Moreover, previous study has revealed that a pooled lysate from at least 1000 cells in any given LCM sample produces a reproducible result no matter where in the tissue the cells were procured, ${ }^{14}$ thus we use the analytical result from LCM as the 'gold standard' for our quantitative tissue analyte measurements.

Using a study set of human colorectal cancer tissue specimens in which LCM was used to procured highly enriched populations of tumor epithelium, stroma within the tumor, along with the patient-matched undissected tissue sample from a contiguous cryostat section, we analyzed the expression or activation (phosphorylation) of large numbers of key signaling proteins using RPMA technology. We found that, expectedly, tumor epithelium and tumor stroma have very different signaling profiles (Figures 1 and 2). This is in keeping with past analysis of prostate cancer ${ }^{21}$ using similar analytical techniques. Previous studies ${ }^{2}$ have shown the dynamic and fundamental role of stromal cells in tumor development due to the cross talk between tumor surrounding microenvironment and cancer epithelia. As well, recent analyses of cellular signaling networks within the tumor microenvironment in colorectal cancer have shown dramatic epithelial-mesenchymal transition of the tumor epithelium signaling, as the tumor and stroma signaling portraits became harder to distinguish the more closer the stroma was to the tumor epithelium. ${ }^{10}$

When these two cellular inputs were compared with the matched undissected material, we found that all three compartments (LCM tumor, LCM stroma and undissected) largely clustered separately using unsupervised analysis (Supplementary Figure S1), which showed that the undissected material generates an entirely different signature than either LCM-procured tumor epithelium or stroma (Figures 1 and 2). Conclusions about which pathways and which signaling molecules are activated in any specific patients' tumor would then be dramatically different if undissected material was the input for the molecular profile. The results confirm that the cellular composition of the specimen is a major source of variability and bias. Moreover, efforts to recapture the LCM signature by mathematically rescaling the data from the undissected material using the percentage of the tumor epithelium present in the originating tissue sample were not successful (Figures 1 and 2), indicating that the LCM profile cannot be empirically determined in a surrogate manner, and must be directly measured. This result is based on the fact that though cancer tissue samples may present with cellular compartments such as tumor epithelium that are determined to be approximately equivalent by a pathologist (for example, all have $50 \%$ tumor 
epithelium), the cellular composition within the sample are highly variable (for example, fibroblasts, immune cells, vascular cells, and so on). Tissue images shown in Figure 5, highlight this issue in which all three different examples of colorectal cancer specimens $(40 \times$ objective $)$ have the same amount of starting tumor epithelium (50\%), yet the presence of tissue infiltrating immune cells, endothelial proliferation, normal appearing epithelial cells, stromal cells and fibers appear very variable in the selected specimens. As most signaling molecules are ubiquitously expressed in nearly every cell type, with activation/phosphorylation of these molecules also variable in every cell type, heterogeneity of the cellular compartments would be expected to have profound effects on measured expression/activation.

Although our analysis focused on the evaluation of the impact of LCM on protein pathway activation determination, previous studies have found gene expression profiling signatures to be similarly biased by lack of cellular-enrichment efforts such as LCM before molecular analysis. Sugiyama et $a l^{23}$ demonstrated the central role of LCM procedure for specimens in which the stromal cells represents $>30 \%$ of the entire tissue. Indeed, in this study, the author highlighted that in bulk cancer samples several genes encoding for immune system proteins were overexpressed and profoundly influenced the final conclusions. Harrell et $a l^{24}$ analyzed the effect of cancer cell enrichment on breast cancer lymph node metastasis analysis. In this study, gene expression profiling of primary breast cancer was compared with matched lymph node metastasis. For each sample, the genomic profile of whole tissue was compared with the one of highly enriched cancer population, isolated by LCM. Only $2 \%$ of the genes identified were differentially expressed in both analyses. Even more remarkable was that merely less than half of them presented the same trend in the undissected and LCM material. A study comparing gene expression between ER-positive and ER-negative human breast tumors found only about one-third of the identified differentially expressed transcripts were common between microdissected and bulk tumor samples. ${ }^{25}$ Wulfkuhle et $a l^{18}$ analyzed the impact of LCM on human breast cancer tissue proteomic profiles. In this study, the profile of whole-tissue lysate and microdissected epithelium was compared in 22 different patients. This study highlighted a profound discordance between the signaling generated by the whole-tissue lysate and LCM material also in samples with a high percentage of cancer epithelium.

A focused analysis of two important protein markers in colorectal tumorigenesis and treatment strategies, COX2 expression and EGFR activation (phosphorylation), revealed profound differences in measurement if LCM was not used to enrich the cellular input for molecular analysis. Recent publications have suggested that measuring phosphorylated EGFR in CRC may be an important biomarker for predicting response to cetuximab. ${ }^{26}$ Comparison of COX2 and phosphorylated EGFR levels in patient tissue specimens that varied between 20 and 60\% tumor showed no correlation between undissected and LCM measurements regardless of the starting tumor content (Figure 3). Suggestions that tumor tissue biomarker analyses that start with samples with highly enriched tumor epithelial cell content could overcome the need to use laborious cell-enrichment techniques for discovery do not seem to support by our data, as tumor specimens with majority of tumor cell content $(60 \%)$ had the same LCM: undissected correlation as tumors with minority tumor epithelium content (20\%) (Figure 3). No trend was seen with either COX2 expression or phosphorylated EGFR values. Even specimens that were matched and segregated for percent tumor composition showed dramatic differences between the rank ordering of the two analytes. The rankorder analysis was used to mimic a clinical situation whereby patient tumor samples are analyzed by molecular profiling to select and stratify patients based on the relative expression levels/activation levels of drug target biomarkers. We found COX2 and phosphorylated EGFR measurements whereby patient's values would have gone from the top quartile of the population to the bottom quartile and vice versa (Figure 4), which would be entirely unacceptable in the clinical or research setting. As we used pooled LCM cells from many separate regions of the tissue section, which has been previously shown to produce reproducible signaling analysis, the results would not be expected to be significantly affected by site of LCM. In fact, as shown in Supplementary Figure S1, the LCM portraits from entirely different patients was more similar to each other than they were to the undissected signatures from the same patient, as the clustering was based on sample input (LCM vs undissected) and not patient. Past results on other pathway biomarkers that are used to stratify patients for therapeutic response such as HER2 in breast cancer also indicated that LCM enrichment before analysis by RPMA was necessary for accurate HER2 protein level determination. $^{18}$

Several recent studies support the requirement of upfront cellular isolation of tumor cells before tissue-based molecular analysis. Giercksky et $a l^{27}$ and Bertheau et $a l^{28}$ demonstrated that the use of microdissection increases the sensitivity in detecting the loss of heterozygosis in breast cancers. They analyzed $\mathrm{LOH}$ in colorectal liver metastasis and breast cancer samples and found a twofold increase in sensitivity of detection of genetic alterations using microdissected material compared with undissected sample input. Moreover, Bertheau et al showed that onethird of breast cancer characterized by loss of heterozygosis would remain undiagnosed without an upfront cellular-enrichment step such as microdissection. The high specificity of the output data obtained with these techniques requires the use of highly enriched cell populations as starting material to avoid misleading results due to the presence of undesired cells.

Taken together, our study seems to support the critical need for a starting sample input of enriched and highly 
purified tissue cells for accurate protein pathway biomarker measurements in colorectal cancer. Although more work needs to be performed on a wider variety of tumor types, on the basis of the data described herein along with previous published findings on the impact of LCM for accurate determination of signaling activation in breast cancer, we recommend the use of LCM or other microdissection techniques for all protein pathway activation analysis and systems biology/network analysis of tumor tissue samples. Companion diagnostic protein biomarkers that provide therapeutic guidance and select patients for the correct therapies must absolutely be accurate in the information they provide to physicians if we are to realize the promise of personalized therapeutics.

Supplementary Information accompanies the paper on the Laboratory Investigation website (http://www.laboratoryinvestigation.org)

\section{ACKNOWLEDGEMENTS}

This work was supported by the Italian Istituto Superiore di Sanità in the framework of the Italy/USA cooperation agreement between the US Department of Health and Human Services, George Mason University, and the Italian Ministry of Public Health, as well as the generous support of the College of Science, George Mason University.

\section{DISCLOSURE/CONFLICT OF INTEREST}

LAL and EFP have stock ownership and are founders in Theranostics Health, Inc, which has licensed techniques described in the paper. VSC, LAL, EFR and MP receive royalties on University-owned patents that cover techniques described herein.

1. Liotta LA, Kohn EC. The microenvironment of the tumor-host interface. Nature 2001;411:375-379.

2. Kalluri R, Zeisberg M. Fibroblasts in cancer. Nat Rev Cancer 2006;6: 392-401.

3. Thiery JP. Epithelial-mesenchymal transitions in tumour progression. Nat Rev Cancer 2002;2:442-454.

4. Gassmann P, Haier J. The tumor cell-host organ interface in the early onset of metastatic organ colonisation. Clin Exp Metastasis 2008;25:171-181.

5. De Wever O, Pauwels P, De Craene B, et al. Molecular and pathological signatures of epithelial-mesenchymal transitions at the cancer invasion front. Histochem Cell Biol 2008;130:481-494.

6. Ng K, Zhu AX. Targeting the epidermal growth factor receptor in metastatic colorectal cancer. Crit Rev Oncol Hematol 2008;65:8-20.

7. Hosmi J, Daud Al. Spectrum of activity and mechanism of action of VEGF/PDGF inhibitors. Cancer Control 2007;14:285-294.

8. Attoub S, Rivat C, Rodrigues $S$, et al. The c-Kit tyrosine kinase inhibitor STI571 for colorectal cancer therapy. Cancer Res 2002;62:4879-4883.

9. Chiang GG, Abraham RT. Targeting the mTOR signaling network in cancer. Trend Mol Med 2007;13:433-442.

10. Sheehan KM, Gulmann C, Eichler GS, et al. Signal pathway profiling of epithelial and stromal compartments of colonic carcinoma reveals epithelial-mesenchymal transition. Oncogene 2008;27:323-331.
11. Wulfkuhle JD, Aquino JA, Calvert VS, et al. Signal pathway profiling of ovarian cancer from human tissue specimens using reverse-phase protein microarrays. Proteomics 2003;3:2085-2090.

12. Cheng L, MacLennan GT, Zhang S, et al. Laser capture microdissection analysis reveals frequent allelic losses in papillary urothelial neoplasm of low malignant potential of the urinary bladder. Cancer 2004;101: 183-188.

13. Miura K, Bowman ED, Simon R, et al. Laser capture microdissection and microarray expression analysis of lung adenocarcinoma reveals tobacco smoking- and prognosis-related molecular profiles. Cancer Res 2002;62:3244-3250.

14. Paweletz CP, Charboneau L, Bichsel VE, et al. Reverse phase protein microarrays which capture disease progression show activation of pro-survival pathways at the cancer invasion front. Oncogene 2001;20:1981-1989.

15. Petricoin III EF, Bichsel VE, Calvert VS, et al. Mapping molecular networks using proteomics: a vision for patient-tailored combination therapy. J Clin Oncol 2005;23:3614-3621.

16. Petricoin EF, Espina V, Araujo RP, et al. Phosphoprotein pathway mapping: Akt/mammalian target of rapamycin activation is negatively associated with childhood rhabdomyosarcoma survival. Cancer Res 2007;67:3431-3440.

17. Pierobon M, Calvert V, Belluco C, et al. Multiplexed cell signaling analysis of metastatic and nonmetastatic colorectal cancer reveals COX2-EGFR signaling activation as a potential prognostic pathway biomarker. Clin Colorectal Cancer 2009;8:110-117.

18. Wulfkuhke JD, Speer R, Pierobon M, et al. Multiplex cell signaling analysis of human breast cancer applications for personalized therapy. J Proteome Res 2008;7:1508-1517.

19. Ornstein DK, Gillespie JW, Paweletz CP, et al. Proteomic analysis of laser capture microdissected human prostate cancer and in vitro prostate cell lines. Electrophoresis 2000;21:2235-2242.

20. Gillespie JW, Best CJ, Bichsel VE, et al. Evaluation of non-formalin tissue fixation for molecular profiling studies. Am J Pathol 2002;160:449-457.

21. Grubb RL, Deng J, Pinto PA, et al. Pathway biomarker profiling of localized and metastatic human prostate cancer reveal metastatic and prognostic signatures. J Proteome Res 2009;8:3044-3054.

22. Gulmann C, Espina V, Petricoin III E, et al. Proteomic analysis of apoptotic pathways reveals prognostic factors in follicular lymphoma. Clin Cancer Res 2005;11:5847-5855.

23. Sugiyama $Y$, Sugiyama $K$, Hirai $Y$, et al. Microdissection is essential for gene expression profiling of clinical resected cancer tissues. Am J Clin Pathol 2002;117:109-116.

24. Harrell JC, Dye WW, Harvell DME, et al. Contaminating cells alter gene signatures in whole organ versus laser capture microdissected tumors: a comparison of experimental breast cancers and their lymph node metastasis. Clin Exp Metastasis 2008;25:81-88.

25. Yang $\mathrm{F}$, Foekens JA, $\mathrm{Yu}$ J, et al. Laser microdissection and microarray analysis of breast tumors reveal ER-a related genes and pathways. Oncogene 2006;25:1413-1419.

26. Valentini AM, Pirrelli M, Caruso ML. EGFR-targeted therapy in colorectal cancer: does immunohistochemistry deserve a role in predicting the response to cetuximab? Curr Opin Mol Ther 2008;10:124-131.

27. Giercksky HE, Thorstensen L, Qvist H, et al. Comparison of genetic changes in frozen biopsies and microdissected archivial meterial from the same colorectal liver metastasis. Diagn Mol Pathol 1997;6:318-325.

28. Bertheau P, Plassa LF, Lerebours F, et al. Allelic loss detection in inflammatory breast cancer: improvement with laser microdissection. Lab Invest 2001;81:1397-1402. 